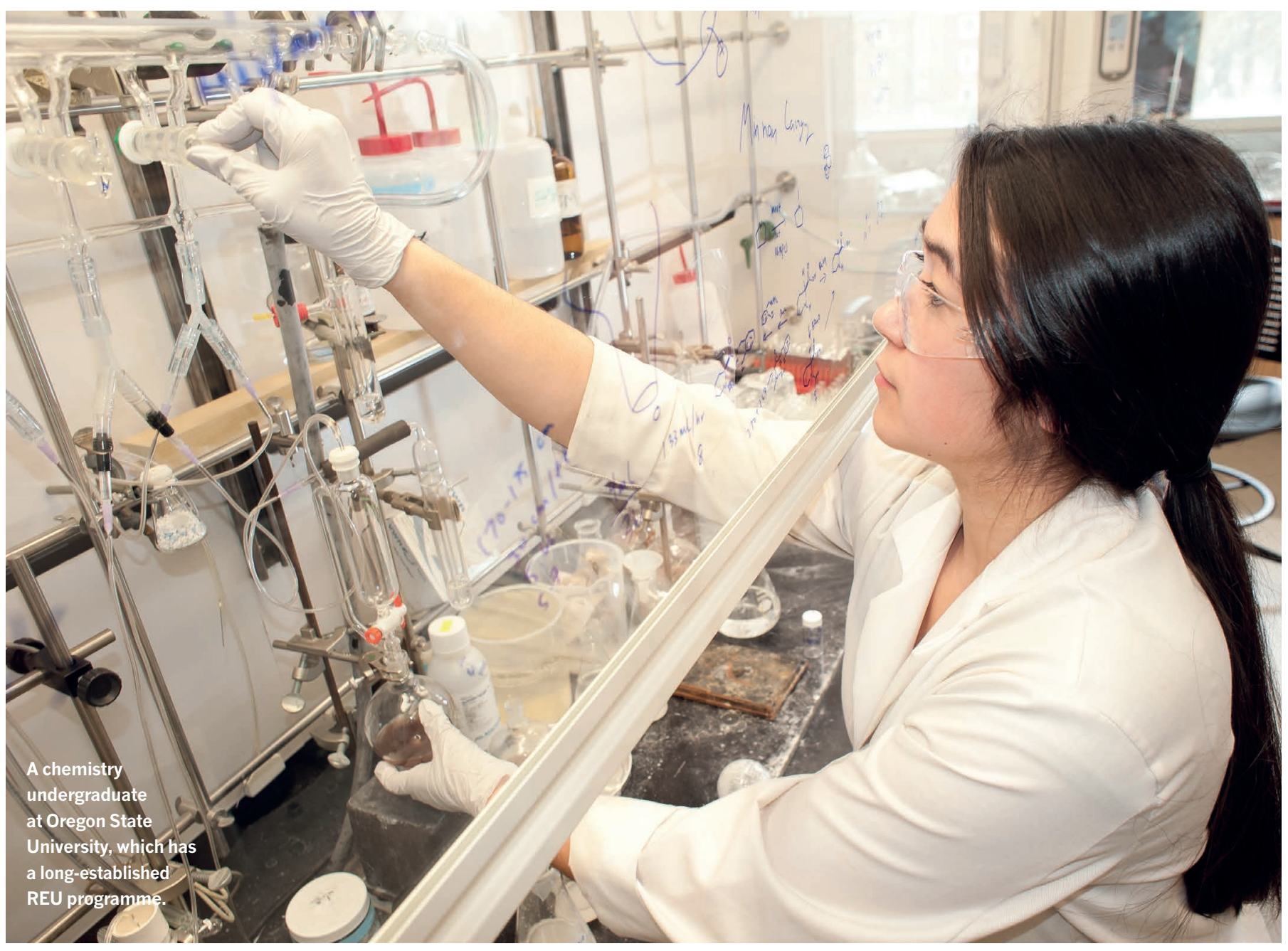

Q\&A

\title{
THE GROUND FLOOR OF DISCOVERY
}

\section{Undergraduate research programmes are expensive, and the benefits can be hard to measure.}

$\mathrm{I}$ $\mathrm{n}$ the early 1950s, universities in the United States began extending research opportunities to undergraduate students. They were supported by the National Science Foundation (NSF), which launched a programme to support undergraduate research in 1958. That programme was cancelled in 1981 but relaunched in 1987 as the Research Experiences for Undergraduates (REU) programme to train the next generation of scientists.
Since then, the REU has awarded almost US $\$ 1.5$ billion to more than 4,500 colleges and university departments. Marine scientist, Itchung Cheung, heads a longstanding REU programme at Oregon State University's Hatfield Marine Science Center. In 2013, the site received more than $\$ 1$ million from the NSF, with co-funding from the Department of Defense, to support 100 students over five years.

Case studies suggest that when undergraduates engage in research, they are more likely to pursue advanced degrees or careers in academic fields. But outcomes have been difficult to measure. In February 2017, the NSF published a study on the effectiveness of such programmes. The committee that wrote the report was chaired by James Gentile, a biochemist and dean emeritus at Hope College in Holland, Michigan.

Gentile and Cheung spoke to Sujata Gupta about the challenges and opportunities of bringing students into the lab. 


\section{Q\&A}

\section{JAMES GENTILE}

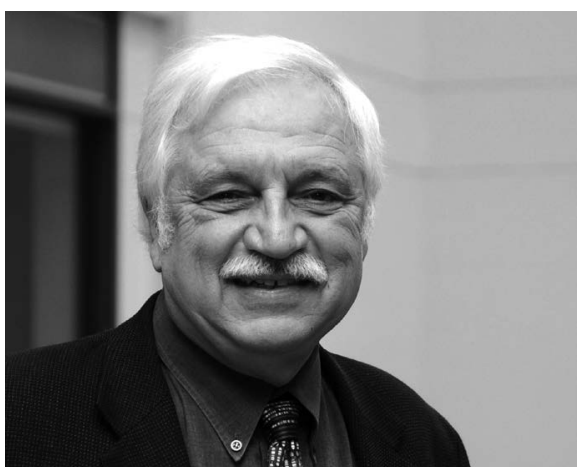

Q: How did the US become a world leader in encouraging undergraduates to conduct research?

A: When I started working at Hope College in 1976, liberal arts colleges were engaging students in undergraduate research, partly because they had no graduate students. The Council on Undergraduate Research (CUR) was founded in 1978 to connect faculty from different institutions. CUR members saw undergraduate research as a way to help students explore science and broaden their own research. Over the years many other institutions have launched undergraduate research programmes, thanks in large part to funding from the NSF and other donors.

\section{Q: How has undergraduate research in the US evolved?}

A: In a classic model, a student joins a faculty member's lab and gets assigned a project that lasts for a summer or longer. The project is sometimes incorporated into the student's classwork during the academic year. With limited faculty and resources, students can't all be handled in this way, so universities have tried to engage students in authentic research through existing courses or by building new courses into the curriculum.

\section{Q: What was the motivation behind the NSF study of the REU programme?}

A: The NSF wanted to quantify the value of undergraduate research, in particular how it aids learning. With undergraduate research, we know students are not just reading and memorizing. These programmes place students on the ground floor of discovery, and give them the opportunity to potentially publish new information. Undergraduate research is the purest form of teaching and learning.

But how do we assess these benefits to justify the high cost of these programmes - hiring one lecturer to teach a class of 200 students is much cheaper than engaging 200 students in undergraduate research. Universities also need to balance the needs of mentors to ensure that they have time to reach their own research goals.
Q: Does undergraduate research encourage more students to pursue science?

A: There's some evidence that students who take research-infused courses consider pursuing a career in a technical field more seriously, or at least electing more science, engineering and math courses, which might move them into a related career.

However, the NSF commission found that there's very little data on this. We recommended that researchers in education design look at the effectiveness of undergraduate research programmes and encourage institutions to collect data on students and their outcomes.

\section{Q: The report indicates that undergraduate research experiences help attract students who are historically underrepresented in science. Can you elaborate?}

A: Many students, including African Americans, Hispanics and Native Americans, have expressed a growing interest in pursuing science and math, but their representation in technical fields and graduate schools has not increased. Case studies indicate that women, minorities, and students who are the first in their family to go to college are more likely to continue in science and technology fields after participating in undergraduate research.
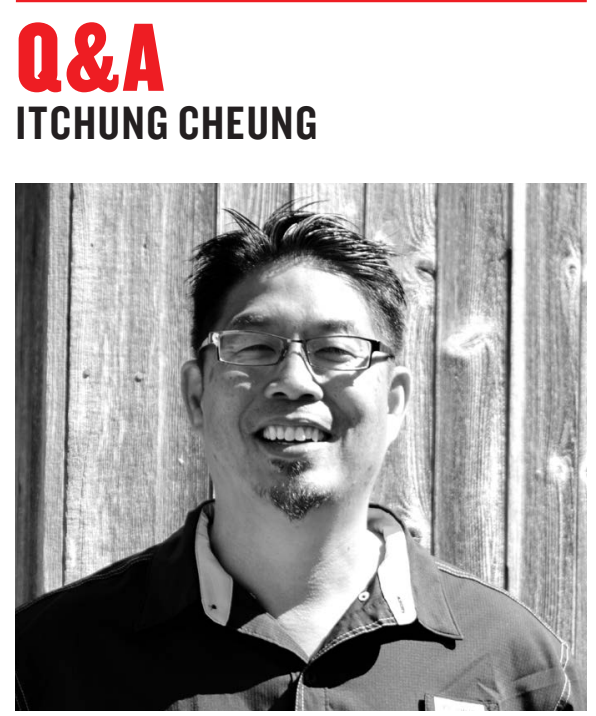

Q: Tell us about Hatfield's REU programme.

A: Our internship programme runs for 10 weeks and includes 27 students this year, which is relatively large compared to other programmes.

Students learn about designing and implementing experiments, analysing data, interpreting the results, and presenting findings in a peer-reviewed publication. We also include training on ethics and the role of research in society, such as discussions about whether it is OK for a scientist to engage in advocacy or how research can have an impact on a community and change policy. So the experience is more than doing research.

\section{Q: How much science do undergraduate students produce?}

A: Our interns present their work at scientific meetings, such as the Association for the Sciences of Limnology and Oceanography Meeting and the American Geophysical Union Meeting, the largest Earth-and space-science meeting in the world. They also publish in peer-reviewed journals. One of our interns recently was one of four authors of a paper on diets and prey availability for juvenile Chinook and coho salmon in northern California.

\section{Q: How do faculty members benefit from the initiative?}

A: Interns look for a question that isn't being addressed by the current projects going on in a researcher's lab. So it's a great opportunity for faculty members to investigate the very edges of their research. Often, faculty lack the funding to pursue that question on their own.

Sometimes, an intern's research leads to a whole new direction for a research group. Several years ago, students found that growth data on long-living bivalves and trees could help to reconstruct temperature variations in the Pacific Ocean over the past several hundred years. Two REU students appeared as authors on the resulting paper. That work also led to a three-year grant from the NSF Paleo Perspectives on Climate Change programme for further research into the region's historic climate patterns.

\section{Q: Do undergraduate research programmes} such as the REU increase diversity in science? A: Yes. The REU provides a stipend, housing, and travel, which helps. But finance isn't the only barrier holding students back.

Students from backgrounds unaccustomed to a culture in which research is a viable career, such as students from community colleges, from low socioeconomic backgrounds, or the first in their family to attend university, tend not to apply because they don't think they'll be selected.

To recruit students, we attend conferences that cater to minorities and we also visit colleges and universities with traditionally underrepresented students. Having diverse representation forces our interns to understand environmental issues from different points of view.

\section{Q: What happens to your students after the programme?}

A: About $80 \%$ of our students continue to graduate school and professional technical positions. Some return to university for advanced degrees. Other students realize that lab life isn't for them, and they go on to a number of other career paths, from teaching to public policy to resource management. As long as they leave with stronger critical thinking skills, that outcome is a success. A student investing many years into graduate training only to realize that field or lab work isn't what they want to do is a considerably worse outcome. 